\title{
Doppler Ultrasound and Fibroscan Parameters Versus Liver Biopsy in Evaluation of Hepatic Fibrosis in Egyptian Patients with Chronic Hepatitis C
}

\author{
Nadia Elwan' ${ }^{1}$, Manal Fathy Hamisa ${ }^{2}$, Mohammed El Hendawy ${ }^{1}$, Shaimaa \\ Soliman $^{\mathbf{3}}$, Nehad Hawash ${ }^{1}$ and Rehab Badawi ${ }^{\mathbf{3}}$ \\ ${ }^{1}$ Department of Tropical Medicine, Faculty of Medicine, Tanta University, Tanta, \\ Egypt. \\ ${ }^{2}$ Department of Diagnostic Radiology, Tanta University, Tanta, Egypt. \\ ${ }^{3}$ Department of Public Health and Community Medicine, Menoufia University Faculty \\ of Medicine, Menoufia, Egypt .
}

Corresponding Author Rehab Badawi

Mobile:

$+201014860506$

E mail:

dr.rb.badawi@gmail. com

Key words: Fibroscan, Hepatitis $C$, Doppler
Background and study aim: Liver biopsy is the gold standard method to assess hepatic inflammation and fibrosis in chronic hepatitis $\mathrm{C}$ infection (HCV). The non-invasive assessment of liver fibrosis is the key target that has inspired many new methods because of the limitations of liver biopsy. The aim of the work was to improve the efficiency of non-invasive liver fibrosis assessment in Egyptian patients with chronic hepatitis $\mathrm{C}$ by comparing Doppler ultrasound (US) of hepatic blood flow and fibroscan with liver biopsy.

Patients and Method: In this retrospective analysis, 78 patients with HCV had already undergone liver biopsies as part of work panel prior to HCV treatment. Fibroscan examination, abdominal ultrasonography and Doppler

\section{INTRODUCTION}

Egypt has the highest incidence of hepatitis $\mathrm{C}$ (HCV) infection worldwide with major public and economic health burden [1]. With persistent liver damage; fibrosis can progress to cirrhosis in up to $15 \%$ to $20 \%$ of cases within 20 years [2]. An estimation of fibrosis progression is critical for assessing the outcome of $\mathrm{HCV}$ infection. The gold standard for assessing the grade of liver fibrosis is liver biopsy, which provides diagnostic information not only on fibrosis but also on many other processes of liver damage, such as necrosis, inflammation, steatosis, copper or iron hepatic deposits [3]. ultrasound were done to the patients by experienced operators.

Results: There was a strong positive correlation between the degree of liver fibrosis by fibroscan and the degree of inflammation in the histopathological analysis. Receiver Operator Characteristic (ROC) curve analysis revealed that fibroscan failed to detect FII fibrosis. However, fibroscan was more accurate in detecting FIII fibrosis.The Doppler ultrasound parameter ROC curve analysis, the portal vein blood flow volume (PVBFV) was shown to be more accurate in detecting lower grades of fibrosis than higher.

Conclusion: For detection higher degrees of fibrosis, Fibroscan has a strong match with liver biopsy; however, Doppler US is more sensitive in detecting lower grades of fibrosis in patients infected with $\mathrm{HCV}$.

Because of its complications, such as bleeding or pain, sampling error can occur in up to $25 \%$ of cases with biopsy perception inter- and intraobserver variability [4] and its limitation in distinguishing between stages F1 and F2 of fibrosis [5] and, in order to assess the severity of liver fibrosis, non-invasive methods have been developed [6]. The non-invasive tools available are either "biological" methods based on serum fibrosis biomarker levels or "physical" methods based on the measurement of liver stiffness using transient elastography [7]. 
Ultrasound-based elastography has led to a breakthrough in the assessment of fibrosis by different methods: one-dimensional ultrasound elastography-transient elastography (TE); or twodimensional ultrasound (or B-mode) using conventional ultrasound imaging-acoustic radiation force (ARFI) imaging or point shear wave elastography (pSWE), real-time 2D shear wave elastography (2D-SWE) and real-time elastography (RTE) [8].

The first ultrasound-based elastography for the diagnosis and staging of liver fibrosis [9] is transient elastography (Fibroscan ${ }^{\circledR}$, Echosens, Paris). By calculation of low- frequency elastic shear wave velocity distributed through the liver, TE uses a mono-dimensional ultrasound to estimate liver stiffness. TE can be accomplished in brief periods and has outstanding (typically less than 5 minutes) intra- and inter- observer variability [10].

Ultrasound is the screening tool for initial assessment of the liver. With the appearance of Doppler ultrasound carried non-invasive means to evaluate secondary changes of blood circulation to chronic liver disease. It could detect the decrease of the portal vein flow velocity and caliber increases, both indicative of portal hypertension [12]. Through the comparison of Doppler ultrasounds of hepatic blood flow and fibroscan with liver biopsy, we aimed to enhance the non-invasive assessment of liver fibrosis in Egyptian patients with chronic hepatitis $\mathrm{C}$.

\section{METHODS}

This was a retrospective comparative observational analysis, with a purposive convenience nonrandomized sample. The informed consent form authorizing the collection of this data was signed by all patients. 250 medical records were reviewed and 78 patients were enrolled with chronic HCV genotype 4 infection. The inclusion criterion included chronic HCV-infected patients aged 18 years or older, male or female, who were assessed for treatment (pegylated interferon and ribavirin) under the national therapeutic regimen.

\section{Exclusion criteria:}

1- Any medical history of compensated or decompensated congestive heart failure to prevent influence of retrograde hepatic blood flow caused by cardiac reflux

2- History of decompensated liver disease

3- Post liver transplantation (to prevent anastomosis affecting blood flow measurement)

\section{4- Pregnancy}

5- Age less than 18 years

6- Hepatitis B virus co-infected patients

We used the free sample size calculator for cohort study at (http://www.openepi.com/ SampleSize/SSCohort.htm) to calculate the sample size for this study. At test power of 0.8 and confidence interval of $95 \%$, sample size was assumed to be 74 patients

Liver biopsy specimens were obtained under complete a septic procedures to retrieve $15 \mathrm{~mm}$ core or at least 15 portal tracts. The specimen was processed and stained with hematoxline and eosine. Fibrosis was tagedona 0 ñ 4scale:F0, no fibrosis; F1, portal fibrosis without septa; F2, portal fibrosis and few septa; F3, numerous septa without cirrhosis; and F4, cirrhosis according to METAVIR scoring system [11].

Liver biopsies were taken from $78 \mathrm{HCV}$ infected Egyptian patients as a part of the workup before HCV treatment. Fibroscan analysis, abdominal ultrasonography, and Doppler ultrasound were performed by a qualified operator who has no data of the patient's clinical history, biochemical examination, or histo pathological results.

All records of the patients are documented in the hospital inpatient and outpatient clinics in the Tropical Medicine Department of Tanta University Hospital, Tanta, Egypt.

All patients were exposed to

- History taking, complete clinical examination, laboratory tests including the full blood analysis, blood urea , serum creatinine the liver function tests (liver enzymes, albumin and bilirubin), prothrombin time, activity, and APRI score were calculated in each patient, and patients were then exposed to:

- Abdominal ultrasonography.

- Transient Elastography (Fibroscan): The hepatic stiffness measurements were conducted by skilled operators following the 
manufacturer's recommendations, using the 502M fibroscan (echosens-France) probe. It was performed with the patient in supine posture and totally abducted by the right arm from the intercostal transthoracic window on the right liver lobe.

The interquartile range (IQR) was considered accurate with a score of less than $30 \%$ and a success rate of not less than $70 \%$. The median value was known to be an indication of the elastic state of the liver. The median value was determined automatically by the software, and the results were expressed in Kilopascals $(\mathrm{kPa})$. All measurements were done with the FibroScan (M) probe after a fasting period of 6 hours. The elastogram score obtained by the device is interpreted through special software to identify the corresponding fibrosis stage in the Metavir score.

- Doppler ultrasound was done by using Toshiba Nemio XG apparatus with a convex probe 3.5 $\mathrm{MHz}$ to assess:

a) the diameter of the portal vein and the volume of the portal vein blood flow

b) the Hepatic venous resistance index (HVRI) for the right hepatic vein

c) The Hepatic artery resistance index (HARI)

d) The Hepatic artery pulsatility index (HAPI)

e) The Splenic artery resistance index (SARI)

The normal diameter of portal vein is highly variable but does not exceed $16 \mathrm{~mm}$ in a resting state on quiet respiration [43]. Normal hepatic artery RI was reported by McNaughton and AbuYousef, to be 0.55-0.7 [44], while normal hepatic pulsatility index was reported by Schneider et al, to be $0.92 \pm 0.1$ [45]. The normal value of splenic artery resistive index (SARI) was reported by Loanitescu to be $0.51+/-0.05$ [46].

\section{Ethical consideration}

Institutional ethical committee approval was obtained before the start of the study. The study protocol conforms to the ethical guidelines of the 1975 Declaration of Helsinki as reflected in a prior approval by the institution's human research committee.

\section{Statistical analysis:}

The data was analyzed using version 2 of Sigma Stat. Quantitative data are presented as mean and
SD while qualitative data are expressed as number and percent. Non-normally distributed data are expressed as range (min-max) and median.

Difference among groups was performed using independent-sample student t-test between groups when normally distributed or ManneWhitney $U$ test if not normally distributed.

The Receiver Operator Characteristic (ROC) curves were developed to assess the accuracy of the diagnosis prediction of fibrosis using version 13.0 of the Social Sciences Statistical Package (SPSS) (IBM Corp, Armonk, NY, USA). The areas of individual tests with $95 \%$ confidence intervals under ROC curves (AUCs) were calculated and compared. The coefficient of Correlation was measured by Spearman's correlation. The findings were found statistically significant when $\mathrm{p}<0.05$ for all used tests.

\section{RESULTS}

This retrospective research was conducted on 78 HCV patients with a mean age of $43.17 \pm 10.77$ years and $37(47.4 \%)$ males. There was no statistical difference in (AST to Platelet Ratio Index) APRI score among patients categorized according to fibrosis degree in liver biopsy (P> 0.05).

Sixty patients had abnormal findings in ultrasound; 20 patients had bright liver, 12 patients had periportal fibrosis, and 28 had ultrasonic findings of liver cirrhosis as coarse echo pattern, irregular outlines, enlarged caudate lobe and shrunken liver in some patients. Regarding splenic examination 16 patients had splenomegaly. The kidney examination in all patients showed normal size and good differentiation between cortex and medulla except for one patient who had grade one nephropathy.

According to fibrosis and activity detected by liver biopsy:

- According to fibrosis degree according to METAVIR scoring system[11]: 57 patients with mild fibrosis (F1 and F2) and 21 patients with significant fibrosis (F3).

- According to activity degree: 35 patients with mild activity (A1) and 43 patients who showed moderate to severe activity (A2-A3) 
In order to evaluate the fibroscan results (figure 1):

There was a statistically significant difference in fibroscan results with higher scores detected in higher fibrosis $\mathrm{P}<0.001$ and more activity (A2 and A3) in liver biopsy $\mathrm{P}<0.001$. The degree of fibrosis in liver biopsies was significantly correlated with liver texture in ultrasound $(\mathrm{r}=0.430, \mathrm{p}=0,00245)$ and fibroscan $(\mathrm{r}=0.51$, $\mathrm{p}<0.001)$. Also, the activity degree showed a significant positive correlation in liver biopsy with fibrosis degree $(r=0.49, \quad p<0.001)$. The ROC curve analysis showed that fibroscan failed to detect FII fibrosis (AUC 0.243, 95\% confidence interval (CI) 0.074-0.411). However, fibroscan was more accurate in detecting F3 fibrosis $(\mathrm{AUC}=0.816,95 \%$ CI $0.666-0.971)$ at cut off values $\geq 7.95 \mathrm{kPa}$ fibroscan had $85.7 \%$ sensitivity and $60.5 \%$ specificity in detecting F3 fibrosis.

The analysis of Doppler ultrasonic parameters of the liver:

The normal diameter of portal vein is highly variable but does not exceed $16 \mathrm{~mm}$ in a resting state on quiet respiration [43].

There was a statistically significant decrease in portal vein flow volume $(\mathrm{P}<0.001)$ in moderate fibrosis in comparison to mild fibrosis also, Portal vein flow volume was decreased significantly in the patients when compared to the average documented values $\mathrm{P}<0.001$, Also a significant negative correlation between fibrosis degree in liver biopsy and portal vein flow volume was found $(\mathrm{r}=-0.55, \mathrm{p}<0.001)$. No statistical differences in splenic artery resistance index (SARI), hepatic artery resistance index (HARI), and hepatic artery pulsatility index (HAPI) $(\mathrm{P}>0.05)$ between the two groups, however, in our patients ,HARI , HAPI , and SARI were significantly higher compared to the average documented values $(\mathrm{p}=0.03,<0.001$ and $<0.001$ respectively). There was no difference in results of HARI, HAPI, SARI, HVRI among patients with mild and moderate activity compared to patients with severe activity in the liver biopsy as shown in cases (figure 3-5).

As regards ROC curve of portal vein blood flow volume for the detection of fibrosis degree in liver biopsy; For F2, AUC was 0.645 (95\% CI $=0.424-0.866)$. At a cut-off point $\leq$ $0.443 \mathrm{~L} / \mathrm{min}$, the portal vein blood flow volume had sensitivity of $76.9 \%$ and specificity of $60 \%$ in detecting F2 hepatic fibrosis in liver biopsy and For F3, it was not an accurate method $(\mathrm{AUC}=0.201,95 \% \mathrm{CI}=0.61$ 0.340 ) figure 2.

Table (1): Comparison between patients activity in liver biopsy and fibroscan elasticity score

\begin{tabular}{|l|c|c|c|c|}
\hline $\begin{array}{c}\text { All patients } \\
(\mathbf{n}=\mathbf{7 8})\end{array}$ & $\begin{array}{c}\text { Mild activity } \\
(\mathbf{A 1}) \\
(\mathbf{n}=\mathbf{3 5})\end{array}$ & $\begin{array}{c}\text { Moderate to severe activity (A2-A3) } \\
(\mathbf{n = 4 3 )}\end{array}$ & $\mathbf{T}$ & $\mathbf{P}$ \\
\hline Range $(\mathrm{KPa})$ & $3.4-10.2$ & $3.8-38$ & & \\
Mean \pm SD & $5.94 \pm 1.98$ & $11.93 \pm 8.18$ & -2.878 & $0.005^{*}$ \\
Median & 5.57 & 8.3 & & \\
\hline
\end{tabular}

Significant $(\mathrm{P}<0.05)$.

Table (2): Portal vein blood flow volume (PVBFV), hepatic artery resistance index (HARI), hepatic artery pulsatility index (HAPI) and splenic artery resistance index (SARI) among our patients in comparison with normally decoumented values

\begin{tabular}{|l|c|c|c|c|}
\hline & Normal range & All patients & t- test & P value \\
\hline $\begin{array}{l}\text { PVBFV }(1 / \mathrm{min}) \\
\text { Mean } \pm \text { SD }\end{array}$ & $0.864 \pm 0.188(13)$ & $0.625 \pm 0.336$ & 4.389 & $<0.001^{*}$ \\
\hline $\begin{array}{l}\mathrm{HARI}\left(\mathrm{cm}^{3} / \mathrm{s}\right) \\
\text { Mean } \pm \mathrm{SD}\end{array}$ & $0.65 \pm 0.1(14)$ & $0.72 \pm 0.12$ & -3.169 & $<0.002^{*}$ \\
\hline $\begin{array}{l}\mathrm{HAPI}\left(\mathrm{cm}^{3} / \mathrm{s}\right) \\
\text { Mean } \pm \mathrm{SD}\end{array}$ & $0.92 \pm 0.1(15)$ & $1.33 \pm 0.42$ & -6.715 & $<0.001^{*}$ \\
\hline $\begin{array}{l}\mathrm{SARI}\left(\mathrm{cm}^{3} / \mathrm{s}\right) \\
\text { Mean } \pm \mathrm{SD}\end{array}$ & $0.51 \pm 0.05(16)$ & $0.664 \pm 0.12$ & -8.684 & $<0.001^{*}$ \\
\hline
\end{tabular}

Significant $(\mathrm{P}<0.05)$. 
Table (3): Comparison between patients with mild and moderate fibrosis in liver biopsy regarding fibroscan elasticity score and Doppler parameters

\begin{tabular}{|c|c|c|c|c|}
\hline & $\begin{array}{l}\text { Mild fibrosis } \\
\quad(\text { FI-FII }) \\
(\mathbf{n}=57)\end{array}$ & $\begin{array}{l}\text { Moderate fibrosis (FIII) } \\
\qquad(\mathbf{n}=\mathbf{2 1})\end{array}$ & $\mathbf{t}$ & $\mathbf{P}$ \\
\hline $\begin{array}{l}\text { Fibroscan score } \\
\text { Range }(\mathrm{KPa}) \\
\text { Mean } \pm \text { SD } \\
\text { Median } \\
\end{array}$ & $\begin{array}{c}3.4-38 \\
8.82 \pm 6.4 \\
7.3 \\
\end{array}$ & $\begin{array}{c}7-33.3 \\
17.34 \pm 9.12 \\
16.8 \\
\end{array}$ & 2.137 & $0.038 *$ \\
\hline $\begin{array}{l}\mathrm{PVD}(\mathrm{mm}) \\
\text { Range } \\
\text { Mean } \pm \mathrm{SD} \\
\text { Median } \\
\end{array}$ & $\begin{array}{c}7.6-20 \\
12.34 \pm 2.54 \\
12.3 \\
\end{array}$ & $\begin{array}{c}9.2-14.8 \\
12.17 \pm 2.12 \\
12 \\
\end{array}$ & 0.161 & 0.868 \\
\hline $\begin{array}{l}\text { PVBF (L/min) } \\
\text { Range } \\
\text { Mean } \pm \text { SD } \\
\text { Median }\end{array}$ & $\begin{array}{c}0.102-1.478 \\
0.675 \pm 0.33 \\
0.64\end{array}$ & $\begin{array}{c}0.051-0.63 \\
0.33 \pm 0.199 \\
0.284\end{array}$ & 2.674 & $0.01 *$ \\
\hline $\begin{array}{l}\text { HARI }\left(\mathrm{cm}^{3} / \mathrm{s}\right) \\
\text { Range } \\
\text { Mean } \pm \text { SD } \\
\text { Median }\end{array}$ & $\begin{array}{c}0.54-0.94 \\
0.732 \pm 0.106 \\
0.73 \\
\end{array}$ & $\begin{array}{c}0.41-0.89 \\
0.657 \pm 0.195 \\
0.62 \\
\end{array}$ & 1.531 & 0.132 \\
\hline $\begin{array}{l}\text { HAPI }\left(\mathrm{cm}^{3} / \mathrm{s}\right) \\
\text { Mean } \pm \text { SD } \\
\text { Range } \\
\text { Median }\end{array}$ & $\begin{array}{c}1.343 \pm 0.412 \\
0.53-2.09 \\
1.33\end{array}$ & $\begin{array}{c}1.263 \pm 0.539 \\
0.59-2.15 \\
1.145\end{array}$ & 0.452 & 0.653 \\
\hline $\begin{array}{l}\text { SARI }\left(\mathrm{cm}^{3} / \mathrm{s}\right) \\
\text { Mean } \pm \text { SD } \\
\text { Range } \\
\text { Median }\end{array}$ & $\begin{array}{c}0.67 \pm 0.12 \\
0.45-0.95 \\
0.65\end{array}$ & $\begin{array}{c}0.65 \pm 0.056 \\
0.59-0.75 \\
0.64 \\
\end{array}$ & 0.380 & 0.705 \\
\hline
\end{tabular}

Significant $(\mathrm{P}<0.05)$

$\mathrm{PVD}=$ Portal vein diameter and $\mathrm{PVBFV}=$ portal vein blood flow volume Hepatic artery resistance index (HARI) Hepatic artery pulsatility index (HAPI)

Splenic artery resistance index (SARI)

Table (4): Correlation between fibrosis degree in liver biopsy and biochemical tests, fibroscan, ultrasound, and Doppler parameters

\begin{tabular}{|l|c|c|}
\hline & $\mathrm{R}$ & $\mathrm{P}$ \\
\hline ALT & 0.077 & 0.593 \\
\hline AST & 0.029 & 0.842 \\
\hline Albumin & -0.267 & 0.061 \\
\hline Fibroscan & 0.284 & $0.045^{*}$ \\
\hline liver texture by ultrasound & 0.430 & $0.002^{*}$ \\
\hline PVD & 0.038 & 0.792 \\
\hline PVBFV & -0.410 & $0.004^{*}$ \\
\hline HARI & -0.146 & 0.322 \\
\hline HAPI & -0.189 & 0.197 \\
\hline HVRI & -0.254 & 0.075 \\
\hline SARI & -0.0123 & 0.932 \\
\hline
\end{tabular}

Significant $(\mathrm{P}<0.05)$

ALT $=$ alanine aminotransferase

AST $=$ aspartate aminotransferase

$\mathrm{PVD}=$ Portal vein diameter and PVBFV= portal vein blood flow volume

Hepatic vein resistance index (HVRI) of right hepatic vein

Hepatic artery resistance index (HARI)

Hepatic artery pulsatility index (HAPI)

Splenic artery resistance index (SARI) 
A

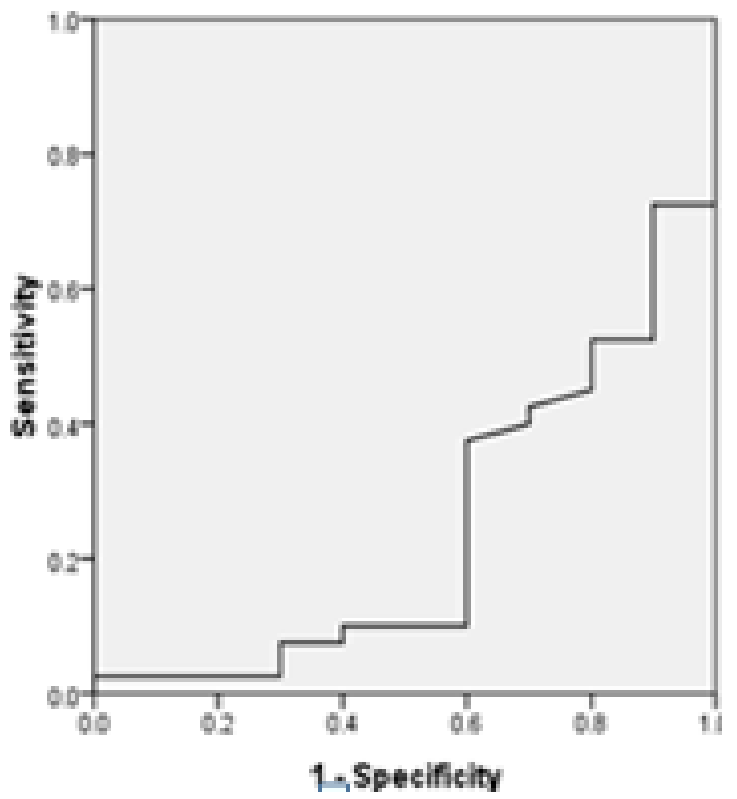

B

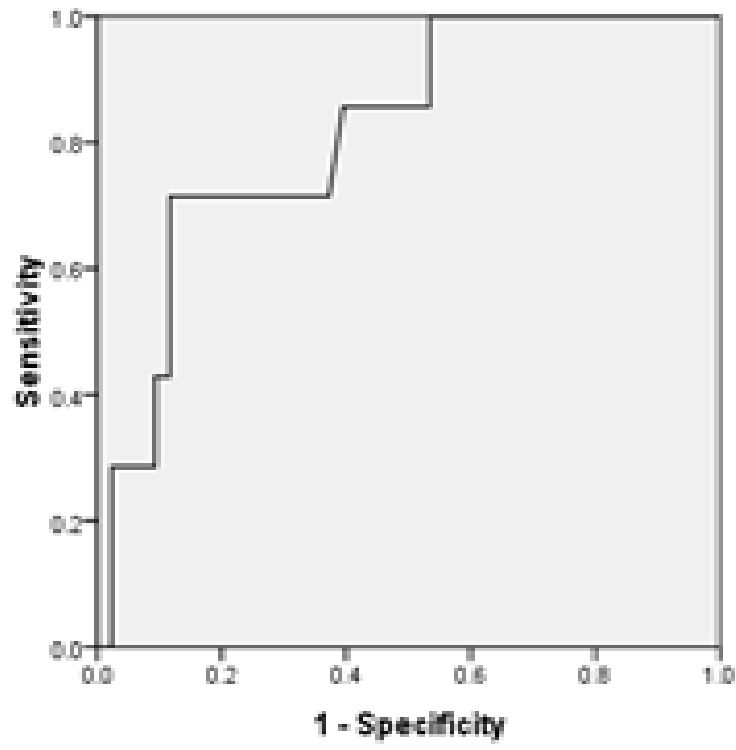

Fig (1): The Receiver Operator Characteristic (ROC) curve of fibroscan to detect fibrosis degree in liver biopsy. (A) For F2, It was not accurate method (AUC=0.243, 95\% CI=0.074-0.411). (B) For F3, AUC was $0.82(95 \% \mathrm{CI}=0.66-0.97)$. At a cut-off point $\geqslant 10.9 \mathrm{kPa}$, fibroscan had sensitivity of $71.4 \%$ and specificity of $83.7 \%$ in detecting F3 hepatic fibrosis in liver biopsy.

A

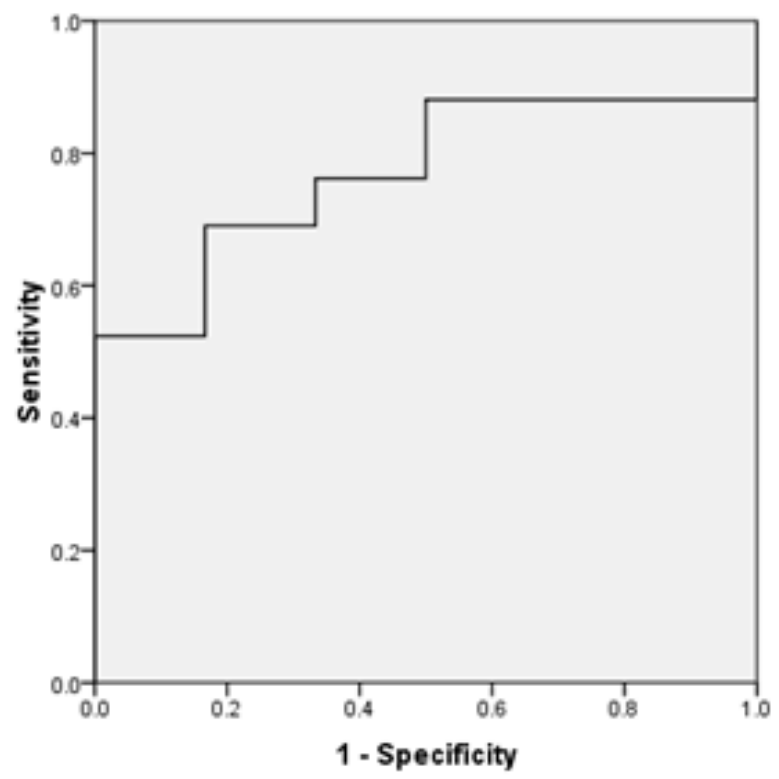

B

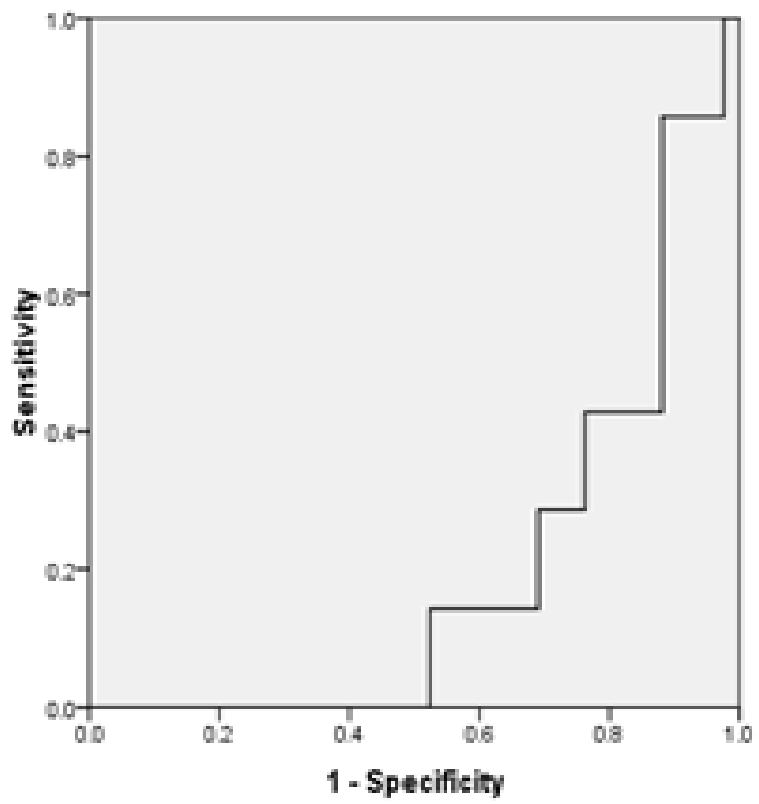

Fig (2): The Receiver Operator Characteristic (ROC) curve of portal vein blood flow volume (PVBF) for detection of fibrosis degree in liver biopsy. (A) For $\leq \mathrm{F} 2$, AUC was 0.77 (95\% CI=0.622-0.917). At a cut-off point $\geq 0.443 \mathrm{~L} / \mathrm{min}$, portal vein blood flow volume had sensitivity of $76.2 \%$ and specificity of $66.7 \%$ in detecting hepatic fibrosis in liver biopsy is F2 or less. (B) For F3, it was not an accurate method $(\mathrm{AUC}=0.201,95 \% \mathrm{CI}=0.61-0.340)$. 

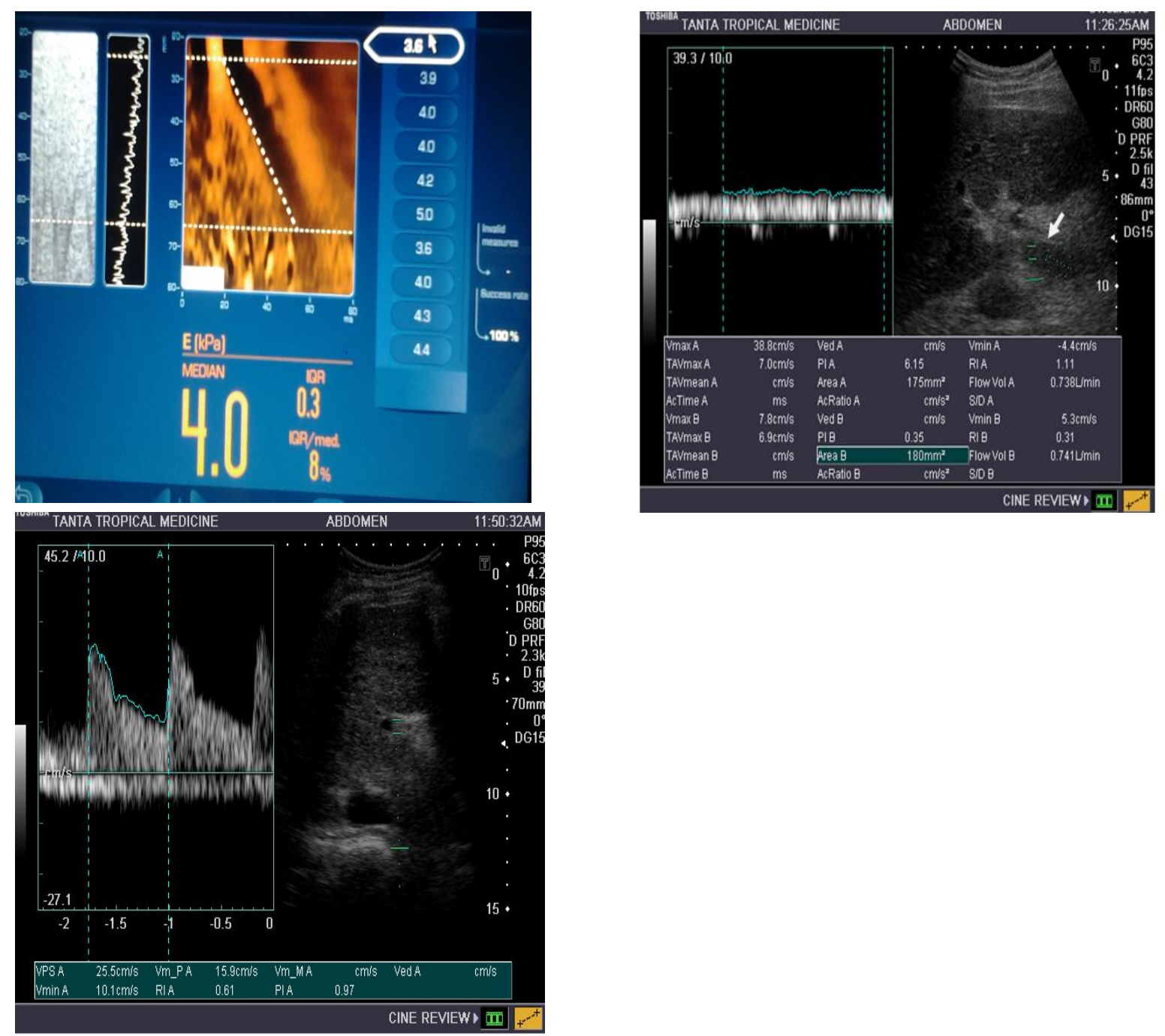

Fig. (3): Case 1 Doppler ultrasound of portal vein showing portal vein blood flow, and hepatic artery (resistive and pulsatility indices) and fibroscan of 32 years old female with F2 in liver biopsy. 

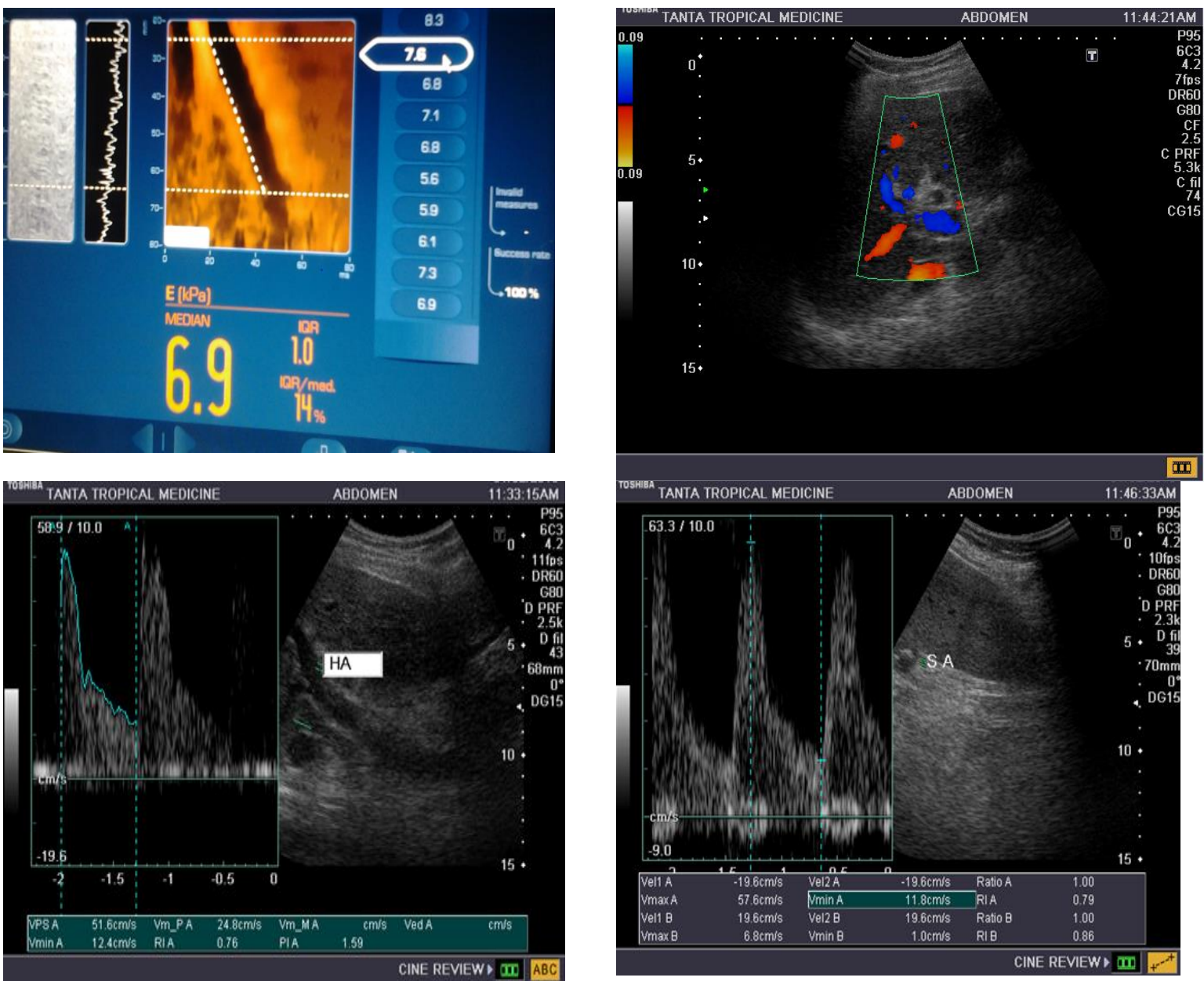

Fig. (4): Case 2: Doppler ultrasound of; portal vein showing hepatopetal flow, hepatic artery resistive and pulsatility indices, splenic artery resistive index and fibroscan of 38 years old male with F2 in liver biopsy.
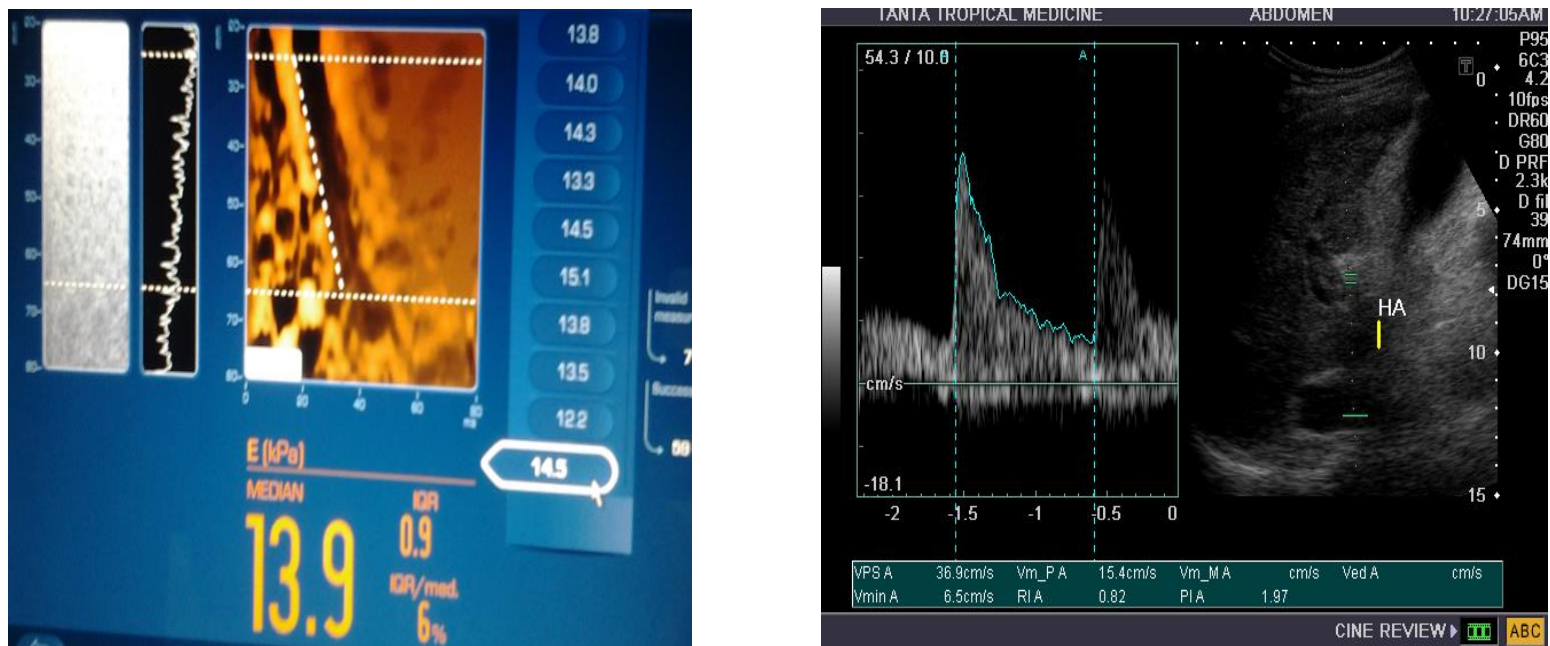

Fig. (5): Case 3: Doppler ultrasound of hepatic artery (resistive and pulsatility indices) and fibroscan of 54 years old female with F3 in liver biopsy. 


\section{DISCUSSION}

In order to improve the efficacy of noninvasive evaluation of liver fibrosis, we compared Doppler ultrasound of hepatic blood flow and fibroscan: with liver biopsy in In Egyptian chronic hepatitis $\mathrm{C}$ patients. In all of the patients who were examined, a statistically significant positive correlation was observed between ultrasound findings and fibrosis score, liver biopsy, and fibroscan results as changes in liver echo pattern and texture were accompanied with increased estimated fibrosis levels in liver biopsy and fibroscan. This was similar to that obtained by Nishiura et al. (2005) who found that the increase of the texture of the liver parenchymal obtained by ultrasound has a statistically significant correlation with the degree of liver fibrosis [17].

Our results were also supported by the results of Abd El Dayem et al, (2013) who showed the diagnosis of hepatic fibrosis, by ultrasonography had $87.5 \%$ sensitivity, $77.5 \%$ specificity, and $84.0 \%$ accuracy, mainly significant fibrosis (F24). But, they concluded that ultrasound imaging cannot identify or precisely diagnose fibrosis in the absence of cirrhosis stigma, like shrunken liver and ascites [18]. It was also in agreement with Davoudi et al, 2015 who found a significant positive correlation between total their gray-scale score and liver fibrosis. They concluded that the liver parenchyma echogenicity may be enough and simple for use in clinical practice to detect fibrosis stage [19] Abd El Maksoud et al. (2015) have also, suggested that echo texture is significantly correlated with the level of fibrosis [20]. Choong et al. (2012), concluded that routine clinical ultrasound is not successful for liver fibrosis staging, giving treatment options, or determining the prognosis of chronic hepatitis secondary to chronic liver disease. They observed, however, that ultrasound is still beneficial for cirrhosis detection [21].

Fibroscan results revealed a strong positive association with the grade of fibrosis and activity index in histopathological analysis. Fibroscan was seemed to be more effective for F3 fibrosis diagnosis. To diagnose F3 fibrosis, we registered a $7.95 \mathrm{kPa}$ fibroscan cut-off value.

Similar findings were obtained by several authors reporting distinct but nearby cutoff values such as Castéra et al, (2005) who reported a cutoff value of 9.5 for fibrosis prediction $\geq \mathrm{F} 3$ with specificity $91 \%$ and sensitivity $73 \%$ [22]. In addition, Ziol et al. (2005) who stated a cutoff value of 9.6 for prediction of fibrosis $\geq$ F3 with specificity $85 \%$ and sensitivity of $86 \%$ [23]. Arena et al. (2008) who put cut off value of $\geq 10.8$ for prediction of fibrosis $\geq \mathrm{F} 3$ with specificity $94 \%$ and sensitivity $91 \%$ [24], This was similar to the results of Fahmy and Badran, (2011) who concluded that: With AUCs 0.92 and 0.95, TE is the most accurate tool for predicting severe fibrosis and cirrhosis and finding that with $\mathrm{TE}$ at $7 \mathrm{kPa}, 86 \%$ were correctly classified for the prediction of significant hepatic fibrosis [25].

Similar to our results, Lutz et al. (2012) ROC analysis showed that in lower fibrosis stages Fibroscan lacks accuracy; and that there was a significant correlation between fibrosis degree detected by fibroscan and inflammation in the histopathological analysis [26]. The positive correlation between liver biopsy and the fibroscan score was also, concluded by Abd El Maksoud et al. (2015), with moderate agreement (matching) between liver biopsy and fibroscan, the lowest matching was in F0 and the highest was in F3 [20]. This was in line with El-Saadany S et al. 2016, who studied fibrosan and biopsy results in $348 \mathrm{CHC}$ patients and found that fibroscan data in moderate fibrosis $(\mathrm{p}<0.001)$ were positively correlated with biopsy, but not mild and no fibrosis $(\mathrm{p}=0.12)$ and concluded that fibroscan was correlated with fibrosis degree in liver biopsy and could be applied as a noninvasive tool for diagnosis moderate (F2- F3), but not mild (F1) fibrosis [27].

We tested Doppler US parameters as an indirect method for grading of hepatic fibrosis. All our patients had continuous hepatopetal blood flow, with a significant negative correlation between PVBF and fibrosis degree in liver biopsy and fibroscan score. These results may be attributed to the increased of hepatic parenchymal resistance that might be caused by fibrosis [28].

In 2002, Vyas et al. analyzed PVBF and PVV and observed that PVBF and PVV in cirrhotic patients were considerably lower than controls [29]. These changes increase as the liver disease gets rising, while Shi et al. (2005) who has observed 38 cirrhosis patients (Child grades A to C) and 20 controls showed that no variation in the diameter of the portal vein is obtained between controls and cirrhotic patients. 
Portal flow for child $\mathrm{C}$ cirrhosis was significantly lower than for Child A and Child B cirrhosis, but no variations of PVBF was found among Child A and $\mathrm{B}$ cirrhosis patients and the controls [30].

On the other hand Walsh et al. (1998) reported that portal vein flow and total hepatic flow were similar in chronic hepatitis $\mathrm{C}$ and controls (The indices of inflammation or degree of hepatic fibrosis were not linked to the state of the liver ). These results may be due to the use of old fashion and inaccurate apparatus [31]. While 36 patients with chronic viral hepatitis, 30 patients without any signs of liver disease as a control group, and 63 patients with cirrhosis with no PVBF difference between three groups were observed by Haktanir et al. (2005), the PVD increased significantly and the mean PVV decreased significantly in cirrhosis compared with hepatitis and control groups [32]. One of the limitations of our study is a few patients with cirrhosis grade 4 . The small sample size limits our ability to detect significant fibrosis if any. Also, the discrepancy between our results and the results obtained from studies that included cirrhotic groups may be due to increased hepatic inflow and decreased PVV mean these changes that accompany portal hypertension.

ROC curve analysis showed that PVB was more accurate in detecting lower grades of fibrosis than higher grades.

Normal hepatic artery RI was reported by McNaughton and Abu-Yousef, to be 0.55-0.7 [44], while normal hepatic pulsatility index was reported by Schneider et al, to be $0.92 \pm 0.1[45]$.

There was a significant increase in mean values of HARI and HAPI in our patients compared to the normally documented values of HARI and HAPI in healthy subjects. These circulatory changes are considered to be occurring in patients with chronic hepatic disease in hepatic arteries are related to the architectural deterioration that occurs within the liver with greater severity of the disease [33].

But there was no difference regarding HARI and HAPI between the two studied groups who had been categorized according to degree of fibrosis by liver biopsy, also there was no difference between HARI and HAPI between the two studied groups who had been categorized according to the degree of fibrosis by fibroscan, and there was no correlation between HARI or HAPI and the degree of inflammation in liver biopsy.

Our Results were similar to those found by Salvatore et al. (2012) who studied 100 patients who had a liver disease caused by hepatitis C, compared results of Doppler parameters with results of fibroscan for detection of the degree of liver stiffness and they found that in these patients HARI rises progressively compared to healthy subjects. They put a cut-off value of 0.64 for HARI at which Patients with LS values above $13 \mathrm{kPa}$ with high sensitivity $(84.4 \%)$ and moderate specificity may be identified $(69.1 \%)$, but finally, they concluded that fibroscan results were more accurate than Doppler parameters despite the direct correlation between HARI and liver stiffness [34].

Although Piscaglia et al. (2001) observed that HARI was higher in patients with severe hepatic fibrosis because the hepatic artery resistance indices seem to be influenced by inflammation and chronic repair that determine hepatic fibrous deposition, and secondly by aging [35]. On the other hand, Lutz et al (2012) observed that HARI did not display any substantial variations in fibrosis scores F1-F3 but was significantly higher in cirrhosis, although no statistically significant differences in HAPI measurements in the various stages of fibrosis (F0-F4/cirrhosis) was found [26]. In comparison, Lim et al. (2005) did not find an association between HARI values and histological scores [36].

The normal value of splenic artery resistive index (SARI) was reported by Loanitescu to be $0.51+/-0.05$ [46].

A significant increase in the mean value of SARI measured among all patients in our study was found compared to normally documented values, this difference may be explained as the advance of hepatic fibrosis contributes to increased portal resistance, which induces increased resistance to splenic artery outflow[37].

But our results did not find any significant differences among the studied groups and there was no correlation between SARI and fibrosis score neither to liver biopsy nor fibroscan results.

Similar to our result Liu et al, (2007) documented that SARI and SAPI were correlated with the degree of fibrosis [38].

Results in this work were different from that obtained by Salvatore et al. (2012) who found that there was a direct correlation between SARI and liver stiffness taking into consideration that 
the study was restricted to the TE values for $\mathrm{F} \leq$ 2. The SARI cutoff value of 0.56 was used to detect patients with LS values $>13 \mathrm{kPa}$ with strong sensitivity (81.3\%) and moderate specificity $(48.5 \%)$. However, they concluded that despite the significant correlation between LS and Doppler US parameters, they were not excellent in detecting liver stiffness [34].

While Cançado et al. (2007) found that the results of SARI were similar in patients with chronic hepatitis and healthy individuals, but it was established that patients with cirrhosis had greater indices [39].

We also measured the hepatic vein resistance index of the right hepatic vein (HVRI) and there was no significant difference between the two studied groups and there was no correlation with fibrosis score in liver biopsy or in fibroscan results ( $p>0.05)$. as far we know, the only study conducted with the estimation of HVRI by Lutz et al. (2012 ) who studied 125 patients (with different grade of fibrosis from F0 to F4) the results showed that HVRI associated with the stage of fibrosis showed the patients without fibrosis exhibited uppermost scores and no significant difference between the different stages of fibrosis was noticed and that HVRI could detect fibrosis of the F2 or higher stages, but it is of limited value in detecting mild fibrosis and that it has superior sensitivity and specificity than fibroscan and HVRI values were not affected significantly by different stages of inflammation or steatosis. This difference may be due to the difference in the study population as they included patients with mixed causes of hepatic cirrhosis while in our result we included only HCV- infected patients who were not cirrhotic [26].

The limitation of the study: this was a retrospective study that leads to losing of some records and data .so numbers of cases in our study were less; and it was a single center study.

\section{CONCLUSION}

Fibroscan has a good matching with liver biopsy in the detection of higher grades of fibrosis and it lacks accuracy in detecting lower grades of fibrosis. Fibroscan results were affected by grade of inflammation in liver biopsy which may cause overestimation of grade of fibrosis. However, Doppler US is more sensitive in detecting lower grades of fibrosis than higher grades in $\mathrm{HCV}$ infected patients.

Funding: None.

Conflict of interest: none.

Ethical approval: available.

\section{REFERENCES}

1. Guerra J, Garenne M, Mohamed MK, Fontanet A. HCV burden of infection in Egypt: results from a nationwide survey. $J$ Viral Hepatitis 2012; 19: 560-567.

2. Lauer $\mathrm{G}$, Walker B. Hepatitis $\mathrm{C}$ virus infection. N Engl J Med 2002; 345 (1): 41 - 52.

3. Sebastiani G, Alberti A. Noninvasive fibrosis biomarkers reduce but not substitute the need for liver biopsy. World J Gastroenterol 2006; 12(23): 3682-3694.

4. Kleiner D, Brunt E, Van Natta M, Behling C, Contos M, Cummings O, et al. Nonalcoholic Steatohepatitis Clinical Research Network. 2005. Design and validation of a histological scoring system for nonalcoholic fatty liver disease. Hepatology 2005; 41: 1313-1321.

5. Poynard T, Lenaour G, Vaillant JC, Capron F, Munteanu M, Eyraud D, et al. Liver biopsy analysis has a low level of performance for diagnosis of intermediate stages of fibrosis. Clin Gastroenterol Hepatol 2012; 10(6): 657-63.e7.

6. Sporea I, Sirli R, Deleanu AE, Popescu A, Focsa M, Danila M, et al. S2084 Transient Elastography (FibroScanR) as Compared toRealTime Elastography (Siemens) in Patients with Chronic Hepatopathies. Gastroenterology 2009; 136(5): A-327.

7. Castera L, Bedossa P. How to assess liver fibrosis in chronic hepatitis $\mathrm{C}$ : serum markers or transient elastography vs. liver biopsy? Liver Int 2011; 31 (1), 13-17.

8. Thiele M, Detlefsen S, Sevelsted Møller L, Madsen BS, Fuglsang Hansen J, Fialla AD, et al. Transient and 2-dimensional shear-wave elastography provide comparable assessment of alcoholic liver fibrosis and cirrhosis. Gastroenterology 2015; 150: 123-133. 10.1053/j.gastro.2015.09.040

9. Sandrin L, Fourquet B, Hasquenoph JM, Yon S, Fournier C, Mal F, et al. Transient elastography: a new noninvasive method for assessment of hepatic fibrosis. Ultrasound Med. Biol. 2003; 29: 1705-1713. 10.1016/j.ultrasmedbio.

10. Boursier J, Konaté A, Gorea G, Reaud S, Quemener E, Oberti F, et al. Reproducibility of liver stiffness measurement by ultrasonographic elastometry. Clin. Gastroenterol. Hepatol 2008; 6: 1263-1269. 10. 
11. Bedossa P. Intra-observer and inter-observer variations in liver biopsy interpretation in patients with chronic hepatitis C. The French METAVIR Cooperative Study Group. Hepatology 1994; 20:15-20.

12. Piscaglia F, Donati G, Serra C, Muratori R, Solmi L, Gaiani S, et al. Value of splanchnic Doppler ultrasound in the diagnosis of portal hypertension. Ultrasound Med Biol 2001; 27 (7), 893-899.

13. Brown H, Halliwell M, Qamar M, Read A, Evans J and Wells P.N. Measurement of normal portal venous blood flow by Doppler ultrasound. Gut 1989; 30 (4): 503-509.

14. McNaughton D, Abu-Yousef M. Doppler US of the Liver Made Simple. RadioGraphics 2011; 31 (1): 161-188.

15. Schneider A, Kalk J, Klein C. Hepatic arterial pulsatility index in cirrhosis: correlation with portal pressure. J Hepatol 1999; 30 (5): 876-881.

16. Loanitescu S, Lliescu L, Harza M, Ismail G, Copaci L. Ultrasound of the spleen (Chapter 8). In EFSUMB Course Book Student Edition (2013). Editors: J. Tuma, R. Badea, and C. F. Dietrich, P23.

17. Nishiura T, Watanabe H, Ito M, Matsuoka Y, Yano K, Daikoku M, et al. Ultrasound evaluation of the fibrosis stage in chronic liver disease by the simultaneous use of low and high frequency probes. Br J Radiol 2005; 78 (927): 189-197

18. Abd El Dayem W, Emam M, Shaheen N, Emara M, Darweish E and Abd El Wahab N. Can combinations of non-invasive parameters replace liver biopsy in the diagnosis of hepatic fibrosis in Egyptian patients with chronic hepatitis C. British Microbiology Research Journal 2013; 3(3): 259-271.

19. Davoudi Y, Layegh P, Sima H, Tatari S, and Faghani R. Diagnostic Value of Conventional and Doppler Ultrasound Findings in Liver Fibrosis in Patients with Chronic Viral Hepatitis. Journal of Medical Ultrasound 2015; 23 (3): 123-128.

20. Abd El Maksoud A, Taha M, El Kassas M, Mahdy R, Mohamed G, Samy H. Prospective comparison of transient elastography and liver biopsy for the assessment of fibrosis in chronic hepatitis $\mathrm{C}$ infection. The Egyptian Journal of Radiology and Nuclear Medicine 2015; 46 (2): 293-297.

21. Choong C, Venkatesh S, Siew E. Accuracy of routine clinical ultrasound for staging of liver fibrosis. J Clin Imaging Sci 2012; 2(3): 58-64.
22. Castéra L, Vergniol J, Foucher J, Le Bail B, Chanteloup E, Haaser M. Prospective comparison of transient elastography, Fibrotest, APRI, and liver biopsy for the assessment of fibrosis in chronic hepatitis C. Gastroenterology $2005 ; 128$ (2): 343-350

23. Ziol M, Handra-Luca A, Kettaneh A, Christidis C, Mal F, Kazemi F. Noninvasive assessment of liver fibrosis by measurement of stiffness in patients with chronic hepatitis C. Hepatology 2005; 41 (1): 48-54.

24. Arena U, Vizzutti F, Abraldes J, Corti G, Stasi C, Moscarella S. Reliability of transient elastography for the diagnosis of advanced fibrosis in chronic hepatitis C. Gut 2008; 57 (9): 1288-1293.

25. Fahmy M, Badran H. Comparison of transient elastography to Doppler indices in prediction of hepatitis $\mathrm{C}$ induced liver fibrosis and cirrhosis. The Egyptian Journal of Radiology and Nuclear Medicine 2011; 42(2): 111-117.

26. Lutz H, Gassler N, Tischendorf F, Trautwein C, and Tischendorf J. Doppler ultrasound of hepatic blood flow for non-invasive evaluation of liver fibrosis compared with liver biopsy and transient elastography. Dig Dis Sci 2012; 57(8): 22222230 .

27. El Saadany S, Soliman H, Ziada DH, Hamisa M, Hefeda M, Selim A, et al. Fibroscan versus liver biopsy in the evaluation of response among the Egyptian HCV infected patients to treatment. The Egyptian Journal of Radiology and Nuclear Medicine 2016; 47: 1-7.

28. Iwao $\mathrm{T}$, Toyonaga $\mathrm{A}$, Oho $\mathrm{K}$, Tayama $\mathrm{C}$, Masumoto H, Sakai T, et al. Value of Doppler ultrasound parameters of portal vein and hepatic artery in the diagnosis of cirrhosis and portal hypertension. Am J Gastroenterol 1997; 92 (6): $1012-1017$

29. Vyas K, Gala B, Sawant P, Das H, Kulhalli P, Mahajan S. Assessment of portal hemodynamics by ultrasound color Doppler and laser Doppler velocimetry in liver cirrhosis. Indian $J$ Gastroenterol 2002; 21 (5): 176-178.

30. Shi B, Wang X, Mu Q, Wu T, Xu J. Value of portal hemodynamics and hypersplenism in cirrhosis staging. World J Gastroenterol 2005; 11 (5): 708-711.

31. Walsh K, Leen E, MacSween R, Morris A. Hepatic blood flow changes in chronic hepatitis C measured by duplex Doppler color sonography: relationship to histological features. Dig Dis Sci 1998; 43(12): 2584-2590.

32. Haktanir A, Cihan B, Celenk C, Cihan S. Value of Doppler Sonography in Assessing the 
Progression of Chronic Viral Hepatitis and in the Diagnosis and Grading of Cirrhosis. $J$ Ultrasound Med 2005; 24 (3): 311-321.

33. Piscaglia F, Gaiani S, Zironi G, Gramantieri L, Casali A, Siringo S, Serra C, et al. Intra- and extrahepatic arterial resistances in chronic hepatitis and liver cirrhosis. Ultrasound Med Biol 1997; 23 (5): 675-682.

34. Salvatore V, Borghi A, Peri E, Colecchia A, Li Bassi S, Montrone L, et al. Relationship between hepatic haemodynamics assessed by Doppler ultrasound and liver stiffness. Digestive and Liver Disease 2012; 44 (2): 154-159.

35. Piscaglia F, Gaiani S, Calderoni D, Donati G, Celli N, Gramantieri L, et al . Influence of liver fibrosis on hepatic artery Doppler resistance index in chronic hepatitis of viral origin. Scand $J$ Gastroenterol 2001; 36 (6): 647-652.

36. Lim A, Patel N, Eckersley R, Kuo Y, Goldin R, Thomas H, et al. Can Doppler sonography grade the severity of hepatitis C-related liver disease? AJR Am J Roentgenol 2005; 184 (6): 1848-1853.

37. Bolognesi M, Sacerdoti D, Merkel C, Gerunda G, Maffei-Faccioli A, Angeli P, et al. Splenic Doppler impedance indices: influence of different portal hemodynamic conditions. Hepatology 1996; 23 (5): 1035-1040.

38. Liu C, Hsu S, Lin J, Hwang U, Liu C, Yang P, et al. Noninvasive Diagnosis of Hepatic Fibrosis in Patients with Chronic Hepatitis C by Splenic Doppler Impedance Index. Clinical Gasteroenterology and Hepatology 2007; 5(10): 1199-1206.
39. Cançado D, Carvalho A, Resende C. Utilization of splenic impedance indices in the evaluation of portal hypertension. Radiol Bras 2007; 40 (3): $155-159$

40. Manning DS, Afdhal NH. Diagnosis and quantitation of fibrosis. Gastroenterology 2008; 134: 1670-81. [PubMed Abstract]

41. Regev A, Berho M, Jeffers LJ, et al. Sampling error and intraobserver variation in liver biopsy in patients with chronic HCV infection. Am J Gastroenterol 2002; 97: 2614-8.

42. Bravo AA, Sheth SG, Chopra S. Liver biopsy. $N$ Engl J Med 2001; 344:495-500.

43. Gorka TS, Gorka W. Doppler sonographic diagnosis of severe portal vein pulsatility in constrictive pericarditis: flow normalization after pericardiectomy. J Clin Ultrasound 1999; 27(2): 84-88.

44. McNaughton DA, Abu-Yousef MM. Doppler US of the Liver Made Simple. RadioGraphics 2011; 31 (1): 161-188.

45. Schneider AW, Kalk JF, Klein CP. Hepatic arterial pulsatility index in cirrhosis: correlation with portal pressure. J Hepatol 1999; 30 (5): 876-81.

46. Loanitescu S, Lliescu L, Harza M, Ismail G, Copaci L. Ultrasound of the spleen. In EFSUMB Course Book Student Edition. Editors: J. Tuma, R. Badea, and C. F. Dietrich. Chapter 8, P23, 2013. 